\title{
Práticas de Saúde e Segurança do Trabalho: Uma Survey em Empresas Brasileiras
}

\section{Exportadoras}

\section{Occupational Safety and Health Practices: A Survey of Brazilian Exporting Companies}

Leonardo Caixeta de Castro Maia ${ }^{1}$, Universidade Federal de Uberlândia, Faculdade de Gestão e Negócios

Daniel Masini Espindola², Universidade Federal de Uberlândia, Curso de Administração

Cristiano Henrique Antonelli da Veiga ${ }^{3}$, Universidade Federal de Uberlândia, Faculdade de Gestão e Negócios

\section{RESUMO}

Este artigo busca debater as práticas de saúde e segurança do trabalho (SST), por meio de uma survey, em empresas brasileiras exportadoras. Para tanto, o artigo apresenta a percepção dos gestores da área SST sobre as práticas operacionais, o ambiente de trabalho, o sistema de segurança e sua conformidade, o nível de desenvolvimento da equipe e, por fim, sobre a cultura organizacional. Concluiu-se que, o principal fator que afeta o grau de amadurecimento de práticas de SST nas empresas está centrado nas certificações. Como limitação, têm-se o número de respondentes. Para futuras pesquisas, a busca de outras estratégias de entrevistas.

Palavras-chave: Práticas operacionais. Saúde e segurança no trabalho. Survey. Manufatura.
Editor Responsável: Prof. Dr. Hermes Moretti Ribeiro da Silva

\section{ABSTRACT}

This article debates the occupational safety and health practices (OSH) through a survey of Brazilian manufacturing companies which export. In this vein, it presents the perceptions of the OSH area managers about operational practices, work environment, safety system and their compliance, the level of team development and organizational culture. It was concluded that the main factor affecting the degree of maturation of OSH practices in companies is centered on certifications. The number of respondents is a limitation of this study. For future research, a search should be made for other interview strategies.

\section{Keywords: Operational practices. Occupational safety and health. Survey.} Manufacture.

1. Avenida João Naves de Ávila, 2121 Bloco 5M, sala 100, Uberlândia, MG; leonardocaixeta@hotmail.com; 2. danielmasini@hotmail.com; 3. chadaveiga@ufu.br

MAIA, L. C. C.; ESPINDOLA, D. M.; VEIGA, C. H. A. Práticas de saúde e segurança do trabalho: uma survey em empresas brasileiras exportadoras. GEPROS. Gestão da Produção, Operações e Sistemas, v. 14, n. 2, p. 108 - 128, 2019. 


\section{INTRODUÇÃO}

Apesar dos avanços ocorridos para a melhoria significativa das condições de trabalho da maioria dos ambientes laborativos e de todos os canais de informação e comunicação, ainda se observam relatos acerca de condições de trabalho degradantes ainda marcantes nesta metade da primeira década do Século XXI, tanto em ambiente nacional (BRASIL, 2014) como internacional (PAGELL et al., 2015; SENRA, 2016).

O número de acidentados no trabalho no Brasil, seja com afastamentos temporários, seja com sequelas permanentes, apesar de apresentar sinais de redução em 2015 de $13 \%$ do número de acidentados no trabalho em relação ao ano anterior, totalizando 612,6 mil casos ao longo de sua série histórica, ainda pode ser considerado muito elevado (BRASIL, 2015).

Destaca-se que, mesmo com essa redução, a quantidade anual média é de aproximadamente 700 mil pessoas acidentadas, gerando um custo de socorro, tratamento e previdenciário de $\mathrm{R} \$ 17$ bilhões por ano para as instituições brasileiras (BRASIL, 2015). Na distribuição por setor de atividade econômica, o setor industrial apresentou 41,09\% do total e o setor de Serviços aparece com 55,69\%, excluídos os dados de atividades consideradas como "ignoradas" (BRASIL, 2015).

Nesse contexto, os estudos acerca das condições de salubridade e segurança do trabalho têm sido marcados por uma avaliação tipicamente unilateral, ou seja, avalia-se com foco centrado apenas nas questões relacionadas aos riscos de acidentes e doenças ocupacionais, deixando para um segundo plano, ou nem sequer são analisadas, as questões que tratam de como o estilo de gestão dos negócios contribuem para esses resultados (MAIA; ESPINDOLA; VEIGA, 2016).

Diante desse cenário, foi estabelecido como objetivo geral do trabalho a identificação de como o sistema de gestão de empresas brasileiras que possuem atuação de exportação desenvolvem suas práticas de gestão e de práticas SST. Nesse sentido, este estudo apresenta a influência dos sistemas de gestão e como esses sistemas influenciam para a obtenção de ambientes de trabalho seguros e saudáveis.

Em se tratando da abordagem referente à temática de modelos de gestão que vêm sendo tipicamente utilizadas pelas organizações, observou-se que, no Japão, desde a década de 1940, e, particularmente, no Brasil, a partir da década de 1990, há vasta referência a sistemas de gestão pela qualidade centrada nos conceitos ISO 9000. De acordo com o Instituto GEPROS. Gestão da Produção, Operações e Sistemas, v. 14, nº 2, p. 108 - 128, 2019. 
Nacional de Metrologia, Qualidade e Tecnologia - Inmetro - (2017), existem, no Brasil, 12.901 empresas certificadas.

Para o desenvolvimento da pesquisa, foi utilizado o método survey realizado por meio de levantamento de dados em empresas brasileiras constantes no cadastro de exportadoras do Ministério de Desenvolvimento, Indústria e Comércio Exterior - MDIC. Utilizou-se como ferramenta para coleta de dados, primeiramente, o contato com os responsáveis pela área de segurança e saúde no trabalho e, posteriormente, realizado o envio do formulário via meio eletrônico.

O artigo encontra-se estruturado em seis partes, iniciando-se pela introdução, seguida da revisão bibliográfica e da metodologia. Posteriormente, são apresentados os resultados da pesquisa, a discussão dos resultados, as derradeiras considerações e finaliza, listando as referências utilizadas. Como contribuição principal, o trabalho apresenta um ineditismo de pesquisa quantitativa acerca das práticas de gestão correlacionadas ao nível de implementação das práticas de saúde e segurança do trabalho em empresas brasileiras exportadoras.

\section{REFERENCIAL TEÓRICO}

A revisão bibliográfica foi dividida da seguinte forma: primeiramente, abordou-se acerca dos objetivos do sistema de gestão de segurança saúde do trabalho. Em seguida, a abordagem se dirige para a necessidade das certificações ISO 9.000 e OHSAS 18.001, perpassando pelos aspectos que tratam sobre o desenvolvimento da equipe e, por fim, da cultura organizacional. A escolha desses tópicos deve-se à influência na definição e padronização das atividades dos processos organizacionais.

\subsection{Sistema de Gestão da Segurança e Saúde do Trabalho}

No Brasil, a legislação pertinente aos aspectos do sistema de gestão da segurança e da saúde no trabalho (SST) é de responsabilidade do Ministério do Trabalho e Emprego (MTE), tenho por base a Consolidação das Leis do Trabalho (CLT) e as ações prevencionistas constantes no Plano Nacional de Segurança e Saúde no Trabalho (BRASIL, 2012). Por meio das Normas Regulamentadoras (NR), previstas pela Portaria 3.214/78 MTE, são estabelecidos os diversos ritos técnicos e administrativos que as empresas devem cumprir com o intuito de manter seus ambientes seguros e saudáveis. 
Assim, em observância à NR 1, denominada Disposições Gerais, é possível identificar as diretrizes administrativas acerca da segurança e saúde no trabalho nas organizações (BRASIL, 2009) e, por meio das demais NR's, são normatizadas as questões técnicas acerca da aplicabilidade do arcabouço legal nas diversas temáticas específicas quanto à SST (BRASIL, 1978).

Alinhados a esse arcabouço legal, muitos gestores buscam um sistema de gestão para além do normativo de maneira que lhes auxiliem no gerenciamento empresarial consorciado de qualidade e de segurança da organização.

As normas trazem embutidas em si uma grande preocupação mundial por melhores condições e resultados no modo como as empresas encaram os impactos que provocam ao meio ambiente e, do mesmo modo, referente à preocupação com a saúde e segurança de seus trabalhadores no ambiente de trabalho, respectivamente (ALMEIDA; NUNES, 2014, p.2).

Os estudos apontam que essa busca se justifica mais por motivos internos, como melhoria da qualidade dos serviços e produtos e qualidade de vida e saúde do trabalhador, do que por motivos externos, como vantagens em marketing e pressões competitivas. Isso se deve a uma mudança das organizações após o acometimento de erros e falhas na implementação da certificação (FENG; TERZIOVSKI; SAMSON, 2008).

Assim, o propósito do sistema de gestão da qualidade, saúde e segurança no trabalho é diminuir os riscos de acidentes e de doenças, bem como favorecer uma imagem boa para as empresas (ALMEIDA; NUNES, 2014), garantir condições para melhorias contínuas e obter uma vantagem competitiva. Os sistemas de qualidade também têm como objetivo fornecer produtos e serviços que atendam melhor às necessidades dos clientes (MAEKAWA; CARVALHO; OLIVEIRA, 2013), enquanto os sistemas de segurança e saúde do trabalho buscam controlar os riscos relacionados às operações e buscar melhorias (VITORELI; CARPINETTI, 2013).

O sistema de gestão usualmente estruturado é aquele com base no ciclo PDCA (Plan, Do, Check, Act), pelo qual se planeja, tendo em vista os clientes e objetivos da empresa, bem como implanta, monitora, mede e executa as melhorias (MAEKAWA; CARVALHO; OLIVEIRA, 2013).

Para Vitoreli e Carpinetti (2013), os sistemas de gestão das organizações devem buscar a integração das normas das certificações. As empresas podem apresentar similaridades entre os certificados ISO 9001 e OHSAS18001 para formação de um sistema de gestão integrado, visto que apresentam passos semelhantes para implementação e GEPROS. Gestão da Produção, Operações e Sistemas, v. 14, nº 2, p. 108 - 128, 2019. 
características de documentação. O objetivo central da integração é diminuir a duplicação de documentos e a redução dos custos. Por outro lado, os seguintes fatores podem influenciar negativamente na integração, tais como, o grau de dificuldade de planejamento, controle e execução das atividades, o tamanho da organização ou o número de funcionários com conhecimento específico.

Outras abordagens gerenciais enfatizam a necessidade de certificação para definir os resultados a serem buscados. A seguir, são apresentados maiores detalhes sobre a certificação ISO 9001 e as suas diretrizes.

\subsection{O sistema de certificação ISO 9001}

A ISO 9001 é responsabilidade da International Organization for Standartization, a qual fornece normas técnicas em nível mundial. A ISO 9001 é uma norma certificável que pode ser utilizada em todos os países e empresas de qualquer tipo e porte, além de ser uma condição essencial para empresas exportadoras de produtos.

Em alguns países, como a Austrália, esse certificado é obrigatório para negociações com organismos governamentais ou companhias privadas. $\mathrm{O}$ ponto chave encontrado nas empresas australianas foi a grande ênfase no planejamento estratégico que as empresas de médio e grande porte dão para alcançar maiores desempenhos operacionais (FENG; TERZIOVSKI; SAMSON, 2008). Os mesmos autores comentam que as empresas de médio e grande porte tendem a ter melhores resultados com a implantação da ISO 9001 do que as empresas de pequeno porte, devido ao fato de conseguirem mais recursos e terem maiores benefícios.

Os benefícios que a certificação ISO pode trazer estão relacionados com as questões gerenciais (como, por exemplo, melhoria do ambiente de trabalho, clareza ao delegar funções) e de eficiência (reduzir custos, aumento de produtividade, redução das paradas, redução de defeitos, por exemplo). Além da busca pela melhoria dos processos em si, os clientes também ficam satisfeitos, pois passam a pagar por produtos que estão sendo desenvolvidos para sua real necessidade (MAEKAWA; CARVALHO; OLIVEIRA, 2013). Além disso, as normas precisam passar por auditorias, apresentar indicadores e metas alcançadas para melhoria contínua do certificado (ALMEIDA; NUNES, 2014).

As empresas que apresentam a certificação cancelada alegam como principal motivo a falta de recursos financeiros. A ausência da certificação impede a contratação de consultoria GEPROS. Gestão da Produção, Operações e Sistemas, v. 14, nº 2, p. 108 - 128, 2019 
especializada para a implantação da ISO 9001, a realização de treinamentos específicos da norma em todos os níveis e a contratação de pessoal especializado que coordene o programa (PINTO; CARVALHO; HO, 2006; MAEKAWA; CARVALHO; OLIVEIRA., 2013). Além disso, não bastam apenas os recursos e o planejamento se os motivos das empresas estão atrelados ao desempenho do negócio, ou seja, desempenho financeiro e de marketing. Assim, os benefícios da ISO 9000 estão relacionados, principalmente, à eficiência de processos e não aos seguintes critérios: custos, motivação dos funcionários ou aumento de participação no mercado (FENG; TERZIOVSKI; SAMSON, 2008).

Outro ponto interessante da certificação ISO 9001 é que ela necessita do comprometimento de todos os membros da organização em todos os estágios de seu processo. Esse é outro problema mencionado pelas pequenas e médias empresas que elas enfrentam na implantação da certificação. Os fatores críticos de sucesso que também são citados pelas organizações para a implementação foram: a identificação dos aspectos filosóficos da qualidade na empresa e treinamento dos funcionários para o planejamento da ISO 9001 (FENG; TERZIOVSKI; SAMSON, 2008).

\subsection{OHSAS 18001}

A norma OHSAS 18001 - Occupacional Health and Safety Assessment Series especifica requisitos para uma gestão das obrigações da empresa com relação à Saúde e Segurança do Trabalho, levando em consideração os requisitos legais, critérios e outras informações. Essa norma pode ser aplicada em empresas de qualquer porte, cultura e geografia, além de ser compatível com a ISO 9001 e ISO 14.001 (ALMEIDA; NUNES, 2014). Por sua vez, Maekawa; Carvalho; Oliveira (2013) afirmam que, segundo a própria OHSAS 18001, o desempenho da gestão de segurança e saúde do trabalho depende dos fatores relacionados ao porte, cultura empresarial, complexidade e natureza dos serviços, produtos e processos de uma empresa.

Sob o ponto de vista do ciclo PDCA, a norma é desenvolvida, inicialmente, por meio do planejamento, com a identificação de perigos ocupacionais, avaliação dos riscos de acidente de trabalho e desenvolvimento de uma política de segurança e saúde ocupacional. Na etapa "fazer", ocorre a implementação das medidas preventivas e treinamento dos funcionários para que haja uma conscientização dos mesmos para o desenvolvimento das tarefas com segurança. A etapa "verificar" corresponde à monitoração das atividades e do GEPROS. Gestão da Produção, Operações e Sistemas, v. 14, nº 2, p. 108 - 128, 2019. 
desempenho do sistema de gestão. Por fim, a etapa de ação corresponde à análise crítica e propostas de melhorias (VITORELI; CARPINETTI, 2013). Ainda de acordo com os autores, a OHSAS foi desenvolvida para preencher uma lacuna representada pela falta de uma norma internacional de segurança e saúde ocupacional, enquanto as normas regulamentadoras do Ministério do Trabalho contribuem para a execução da segunda etapa do ciclo.

\subsection{Desenvolvimento da equipe}

Segundo Gittel e Douglas (2012), a coordenação relacional é um dos três processos gerenciais de inter-relação recíproca entre os trabalhadores, trabalhador/cliente e trabalhador/gerente. Os trabalhadores praticam funções complementares quando estão divididos horizontalmente no trabalho, mas cada um possui um processo de entendimento diferente sobre uma situação e, da mesma forma, tem a sua habilidade para responder a ela. Para solucionar esse problema, algumas escolas apresentaram modelos para alcançar a coordenação relacional e reduzir custos com ferramentas mais mecanizadas e burocráticas. A intenção é que o trabalhador tenha recursos, possibilitando a ele ter tarefas mais independentes e resilientes às mudanças imprevistas no ambiente de trabalho.

Esses autores afirmam que a noção de coordenação deve se apresentar aos seus participantes como uma mente coletiva, reforçando a comunicação e, consequentemente, a reinterpretação do ambiente pelos seus membros, sendo o conhecimento compartilhado o ponto chave para esse processo. Em casos práticos, levar os trabalhadores a aumentarem a atenção ao outro, principalmente, em situações emergenciais, contribui para a criação de uma mente coletiva (BROWN, 1996; PAGELL et al., 2015).

\subsection{A cultura}

Segundo Power et al. (2015), os investimentos e reinvestimentos em práticas de segurança e meio ambiente são maiores em empresas internacionais e de grande porte. Isso se deve ao fato de maiores acessos a recursos e produção em grande escala. O estudo realizado em 24 países demonstrou que, quanto maior for a produtividade da empresa, maior é o seu potencial para reinvestir nessas práticas, porém isso não está ligado com o potencial em investir mais do que a média. Empresas de países que são fortes economicamente tendem a ter maior poder para investir em áreas que não geram lucro propriamente dito. Essas empresas também tendem a ser fortes economicamente devido ao fato de olharem os investimentos em GEPROS. Gestão da Produção, Operações e Sistemas, v. 14, nº 2, p. 108 - 128, 2019. 
práticas de segurança e meio ambiente como algo periférico às operações centrais da organização.

O mesmo estudo ainda demostrou que a média de investimentos em práticas de segurança e meio ambiente das empresas brasileiras ficaram muito abaixo se comparadas com as dos demais países pesquisados. Outro dado apresentado foi que a alta gerência das empresas brasileiras considera menos importante a segurança e o meio ambiente em um mercado competitivo do que empresas de outros países.

No entanto, existem empresas brasileiras que utilizam e incentivam programas voltados para saúde, segurança e meio ambiente. Para essas organizações, investir no bemestar e no trabalho seguro dos funcionários é um recurso que gera vantagem competitiva para elas, conquistando, assim, um recurso raro, que é o seu capital humano. Esse diferencial encontrado em uma empresa pode ser representado pelo clima organizacional que considera as questões de segurança, saúde e bem-estar das pessoas como fatores determinantes para a manutenção e, até mesmo, alavancagem dos resultados (FAVRETTO; ROMAN; SEHNEM, 2016; FILIPPE, 2016).

Segundo Paz e Kipper (2016), as empresas com melhores práticas sustentáveis são aquelas que conseguem integrar os seus colaboradores e a sociedade, alinhando os valores dessa última com os dos primeiros, formando, assim, uma percepção dos indivíduos sobre os resultados de suas ações e convergindo-os para o mesmo pensamento.

Power et al. (2015) afirmam que as características de uma cultura nacional são fatores contingenciais importantes que afetam as decisões gerenciais para investimentos em práticas voltadas para a segurança e o meio ambiente. Os empregados que não são estimulados pelos gerentes por meio de políticas de segurança, saúde, ética e responsabilidade social tendem a adiar tais práticas, encorajar comportamentos no trabalho e evitar procedimentos ou equipamento de segurança. Outros estudos afirmam que o interesse gerencial em segurança e meio ambiente apenas ocorre com o alinhamento dos valores individuais e os da organização.

Para Pagell et al. (2014), a falta de investimentos para a percepção da cultura do trabalhador eleva os riscos em situações de emergências, devendo o mesmo decidir entre produtividade e a segurança. $\mathrm{O}$ próprio trabalhador é um agente da cultura que influencia o ambiente, podendo colocar a empresa em risco caso não haja um sistema controlado coletivamente pelos seus gerentes. 


\section{MATERIAL E MÉTODOS DA PESQUISA}

\subsection{Desenvolvimento do instrumento de pesquisa}

Primeiramente, foi realizada a tradução do instrumento de coleta de dados utilizado no trabalho de Pagell et al. (2015) do inglês para o português. Posteriormente, realizou-se uma revisão de adequação dos termos para o cenário nacional, sendo esse trabalho realizado por dois professores especialistas na área de operações e produção. Para avaliar a sua aplicabilidade, foi feito um pré-teste com dois gestores que exercem atividades na área de segurança, saúde e meio ambiente em suas respectivas empresas, sendo ambas multinacionais de grande porte (COOPER; SCHINDLER, 2011).

\subsection{Definição da amostra}

Com a utilização de dados das empresas brasileiras exportadoras de 2015, conforme relação elaborada pelo Ministério do Desenvolvimento, Indústria e Comércio Exterior (MDIC, 2016), encontrou-se uma população de 23.548 organizações. O primeiro recorte foi quanto ao critério de faturamento, levando-se em consideração apenas empresas com valor de exportação maior do que US\$ 1 milhão, resultando em 6.164 empresas ou 26,17\% da população.

A determinação do tamanho mínimo da amostra para o cálculo da proporção da população foi feita com nível de confiança equivalente a 95\% e erro máximo desejado de 10\%. A amostra corrigida pela população foi de 96 empresas.

A estratificação foi feita a partir da proporção de cada Estado da federação, apresentando São Paulo o maior número de empresas (34\% da amostra). Para que houvesse pelo menos uma representação de todos os estados, foi estabelecido, no mínimo, uma empresa para cada um deles, totalizando, assim, 114 empresas.

\subsection{Validação do questionário}

Para a validação do instrumento, foi realizada uma visita técnica e aplicação-teste do questionário em uma unidade de uma empresa multinacional de grande porte localizada na cidade de Uberlândia, MG e pertencente à lista do Ministério de Desenvolvimento, Indústria e Comércio (MDIC, 2016). Ademais, foram observados o tempo de resposta total e as dúvidas frequentes do profissional da área de segurança que aceitou responder o questionário e, 
posteriormente, comentar pessoalmente as suas percepções sobre o instrumento de pesquisa. A visita também contribuiu para observações no ambiente de trabalho da empresa, sua rotina e aspectos organizacionais individuais quanto ao desenvolvimento das práticas de segurança e saúde no trabalho. Depois dos ajustes necessários, foi enviado, via e-mail, um segundo questionário para se verificar o entendimento do questionário, conforme processo de envio previamente estabelecido.

O questionário foi dividido em três etapas: caracterização do respondente, caracterização da empresa e acerca da utilização das práticas de segurança e saúde do trabalho. Quanto à terceira etapa, foi utilizada a escala Likert de 1 a 7, sendo 1 para 'nunca', 4 para informar que a frequência é 'às vezes' e 7 para declarar que 'sempre' é realizada.

\subsection{Modelo teórico}

O modelo teórico proposto apresenta os seguintes constructos referentes aos conjuntos de práticas adotadas pelos gerentes de acordo com os teóricos e validado pelos especialistas:

- Práticas Operacionais: referem-se a todas as atividades que promovam maior estabilidade dentro das organizações e estão vinculadas à redução das incertezas operacionais na busca pela padronização de processos, aumento da confiabilidade de equipamentos e, consequentemente, redução das quebras e falhas (FLYNN; SAKAKIBARA; SCHROEDER, 1995; PAGELL et al., 2015);

- Ambiente seguro e saudável de trabalho: está relacionado ao aumento da consciência sobre a importância da qualidade da saúde do trabalhador e sua relação com os resultados operacionais, utilizando-se ferramentas que priorizem a segurança e a saúde do trabalhador nas mudanças organizacionais. Nesse sentido, a busca pela maior qualidade tem efeitos na segurança e no bem-estar do trabalhador (BROWN, 1996);

- Sistema de segurança e conformidade: relaciona-se à conformidade do comportamento dos trabalhadores com os procedimentos, metas e regras de segurança que são estabelecidos, monitorados e revisados pela companhia (HAYES et al., 1998; SHAFIQ; KLASSEN; JOHNSON, 2014);

- O desenvolvimento da equipe de trabalho: busca evidenciar os investimentos em treinamentos, seleção e retenção de empregados e está relacionado com os seguintes aspectos: habilidade dos empregados em resolver problemas, coordenação do trabalho, no e entre os diversos departamentos de uma organização, desenvolvimento da capacidade de GEPROS. Gestão da Produção, Operações e Sistemas, v. 14, nº 2, p. 108 - 128, 2019. 
decisão em situações imprevistas ou emergenciais e cumprimento do sistema de segurança e saúde do trabalho (FLYNN; SAKAKIBARA; SCHROEDER, 1995).

- A cultura: é a incorporação e compartilhamento dos pressupostos, valores e processos (visíveis e invisíveis) relacionados à segurança e saúde no trabalho em cada nível organizacional (PAGELL et al., 2014; PAGELL et al., 2015; WU et al., 2010).

\section{ANÁLISE DOS DADOS E RESULTADOS}

Os resultados a seguir apresentados são oriundos das análises dos questionários válidos para esta pesquisa. Para realização da análise dos dados, foram utilizadas as médias aritméticas dos constructos, a estatística descritiva e a aplicação dos testes: Kruskal Wallis, para a análise de variância de dados não paramétricos, Alfa de Cronbach, para análise da confiabilidade, o teste Kolmogorov e Smirnov $(K-S)$, para avaliação da distribuição de dados normais, e a correlação entre as variáveis por meio de teste Pearson.

\subsection{Quanto ao perfil dos respondentes}

$\mathrm{O}$ perfil dos respondentes apresentou uma maioria do gênero masculino, na faixa etária entre 26 a 45 anos, com experiência entre 6 a 15 anos. A diferença de experiências demonstra uma diversidade das respostas, possibilitando abranger aqueles profissionais que adquiriram maiores habilidades em inserir e desenvolver novas práticas e aqueles que estão aprendendo com a empresa por meio de tentativa e erros.

O departamento de atuação dos respondentes, conforme nomenclaturas internas das organizações, foi, em sua grande maioria, da área de Segurança do Trabalho. Alguns responsáveis também afirmaram ser da área de Serviço Especializado em Engenharia de Segurança e em Medicina do Trabalho (SESMT), de recursos humanos e de outros relacionados à indústria e ao meio ambiente.

\subsection{Quanto às informações das empresas}

Quanto às informações das empresas, pode ser observada uma maior quantidade delas no setor primário, principalmente, na agricultura, pecuária, produção florestal e pesca, conforme ilustrado na Tabela 1. Esse setor apresentou 11 empresas, enquanto o setor secundário apresentou 9, além de 1 empresa do setor terciário. 
Tabela 1 - Setores das empresas

\begin{tabular}{lcc}
\hline Descrição & Frequência & Percentual \\
\hline Agricultura, Pecuária, Produção Florestal, Pesca e Agricultura & 8 & 38,1 \\
Indústrias de Transformação & 4 & 19,0 \\
Alojamento E Alimentação & 1 & 4,8 \\
Comércio Atacadista de Café em Grão & 1 & 4,8 \\
Eletroeletrônico & 1 & 4,8 \\
Fabricação de Adubos & 1 & 4,8 \\
Fabricação de Chapas Compensadas e Laminadas & 1 & 4,8 \\
Indústria e Comércio de Óleos Essenciais & 1 & 4,8 \\
Industria, Extração e Refino de Óleos, Biodiesel & 1 & 4,8 \\
Madeireira (compensados) & 1 & 4,8 \\
Petróleo e Gás & 1 & 4,8 \\
Total & 21 & 100,0 \\
\hline
\end{tabular}

Fonte: Pesquisa de campo dos autores (2016).

Na Tabela 2, são apresentados os estados onde as empresas se encontram localizadas. Observa-se que mais de 50\% delas se encontram na Região Sul e Sudeste, de acordo com a proporção por região estabelecida na distribuição da amostra.

Tabela 2 - Estado de Origem

\begin{tabular}{lcc}
\hline Localização da unidade entrevistada & Frequência & Percentual \\
\hline Sem resposta & 5 & 23,8 \\
Minas Gerais & 3 & 14,3 \\
São Paulo & 3 & 14,3 \\
Paraná & 2 & 9,5 \\
Rio Grande do Sul & 2 & 9,5 \\
Alagoas & 1 & 4,8 \\
Amazonas & 1 & 4,8 \\
Goiás & 1 & 4,8 \\
Mato Grosso & 1 & 4,8 \\
Rondônia & 1 & 4,8 \\
Santa Catarina & 1 & 4,8 \\
\hline Total & $\mathbf{2 1}$ & $\mathbf{1 0 0 , 0}$ \\
\hline
\end{tabular}

Fonte: Pesquisa de campo dos autores (2016).

No instrumento de pesquisa, abordou-se sobre o grau de investimentos relacionado ao potencial das empresas em investir em equipamentos de segurança e saúde com relação aos seus concorrentes.

Assim, como resultado, a maioria das empresas da amostra apresentou um grau de investimento na média ou maior que o de seus concorrentes, o que significa uma expectativa maior dos respondentes quanto à realidade das empresas brasileiras. 
Práticas de Saúde e Segurança do Trabalho: Uma Survey em Empresas Brasileiras Exportadoras

\subsection{Quanto à utilização de normas e diretrizes de qualidade}

A ISO 9001 apresentou um índice de implementação de 47\% das empresas da amostra analisada. Os resultados ficaram abaixo do esperado para as empresas exportadoras brasileiras, tendo em vista o discurso de que a norma é considerada uma condição essencial para as mesmas obterem vendas no mercado exterior.

No que trata das questões referentes à implantação da norma ISO 14.001, observou-se que 47,6\% dos respondentes afirmam que essa ação não é considerada, 42,9\% citam a existência de planos para futura consideração e apenas 9,5\% asseveram que já implementaram. Em relação à OHSAS 18.001, verificou-se 52,4\% dizem que sua implantação não é considerada, $14,3 \%$ têm a implantação concluída e outros $14,4 \%$ consideram a futura implantação.

\section{CONSTRUCTOS AVALIADOS}

\subsection{Estatística descritiva}

A Tabela 3 apresenta a Média das respostas para os cinco constructos elaborados para esta pesquisa. Dessa forma, foi calculada a média de cada constructo para cada respondente e, em seguida, a média de todas as respostas, com um total de 21 respondentes. Os constructos que obtiveram maiores desempenhos foram: Sistema de Segurança e sua Conformidade e Práticas Operacionais.

Tabela 3 - Estatística descritiva dos constructos

\begin{tabular}{lllll}
\hline Média do Constructo & Média & Desvio Padrão & Mínimo & Máximo \\
\hline ASST & 5,91 & 1,2026 & 3,20 & 7,00 \\
SSC & 6,21 & 0,9777 & 3,67 & 7,00 \\
CULT & 5,97 & 1,0750 & 3,00 & 7,00 \\
EQUI & 5,73 & 1,2632 & 3,33 & 7,00 \\
PRAT & 6,18 & 0,9314 & 3,20 & 7,00 \\
\hline
\end{tabular}

Fonte: Pesquisa de campo dos autores (2016). Legenda: ASST: Média do constructo "Ambiente seguro e saudável do trabalho"; SSC: Média do constructo "Sistema de segurança e sua conformidade"; CULT: Média do constructo "Cultura"; EQUI: Média do constructo "Desenvolvimento da equipe de trabalho"; PRAT: Média do constructo "Práticas Operacionais".

\subsection{Avaliação de variáveis moderadoras}

A partir da utilização do método Kruskal Wallis, observa-se um resultado significativo entre os constructos e a variável moderadora ISO 9001. Todos os constructos obtiveram 
Práticas de Saúde e Segurança do Trabalho: Uma Survey em Empresas Brasileiras Exportadoras

diferentes desempenhos com relação à implantação ou não da certificação, com exceção do constructo "Cultura", que apresentou um alfa maior do que 5\%, conforme Tabela 4.

Tabela 4 - Avaliação da variável moderadora ISO 9001. (grau de liberdade =1)

\begin{tabular}{llllll}
\hline Média & ASST & SSC & CULT & EQUI & PRAT \\
\hline Qui-quadrado & 4,818 & 8,621 & 3,589 & 8,501 & 3,965 \\
p-value & 0,028 & 0,003 & 0,058 & 0,004 & 0,046 \\
\hline
\end{tabular}

Fonte: Pesquisa de campo dos autores (2016).

Também foi aplicado o método com as médias dos constructos e as demais categorias: Regiões (Norte, Centro-Oeste, Sudeste, Nordeste e Sul), porte da empresa (grande, média, pequena) e fluxo de produção (produção contínua, por projeto, por lote ou batelada), não apresentando resultados significativos ou alfa maior do que $5 \%$.

No que trata das categorias referentes à ISO 14.000 e à OSHAS 18.001, essas não apresentaram dados suficientes para uma análise estatística significativa devido à baixa implementação dessas sistemáticas normativas pelas empresas dos respondentes.

\subsection{Quanto aos pressupostos de estatística multivariada}

\subsubsection{Quanto à confiabilidade interna}

O teste de confiabilidade foi feito a partir do cálculo do Alfa de Cronbach, sendo considerado o valor ideal aquele maior que 0,7 para cada constructo analisado (HAIR et al., 1998), conforme Tabela 5.

Tabela 5 - Análise dos constructos por meio do cálculo de Alfa de Cronbach

\begin{tabular}{llccc}
\hline Ref & Variável Latente & $\begin{array}{c}\text { Número de } \\
\text { indicadores }\end{array}$ & $\begin{array}{c}\text { Alfa de } \\
\text { Cronbach }\end{array}$ & $\begin{array}{c}\text { Alfa de Cronbach } \\
\text { pós purificação }\end{array}$ \\
\hline 1 & Práticas Operacionais & 5 & 0,800 & 0,811 \\
2 & Ambiente Seguro e Saudável do trabalho & 5 & 0,910 & - - \\
3 & Sistema de segurança e sua conformidade & 6 & 0,857 & 0,892 \\
4 & Desenvolvimento da equipe de trabalho & 3 & 0,847 & - - \\
5 & Cultura & 4 & 0,845 & - - \\
\hline & Total & $\mathbf{2 3}$ & &
\end{tabular}

Fonte: Pesquisa de campo dos autores (2016). 
Conforme ilustrado na Tabela 5, todos os constructos estabelecidos para a pesquisa apresentaram Alfa de Cronbach maior do que o valor considerado como referência. $\mathrm{O}$ que mais apresentou confiabilidade foi o constructo relacionado ao "Ambiente Seguro e Saudável do Trabalho".

Observou-se ainda que alguns indicadores poderiam ser descartados em vista do contexto, como, por exemplo, o indicador "Esclarecemos sobre a utilização de Equipamentos de Proteção Individual (EPI's) em áreas de perigo", visto ser essa uma prática normal e usualmente utilizada nas empresas que visam à segurança e à saúde. Depois da retirada desse item, o alfa de Cronbach, após a purificação do constructo "Sistema de segurança e sua conformidade", passou de 0,857 para 0,892 , fato que permite inferir que o uso de EPIs não influencia, estatisticamente, para a formação da cultura em SST nas empresas pesquisadas. Evidencia-se também um número mínimo de três questões para cada constructo latente (KLINE, 2011).

O outro indicador eliminado foi: "Nós buscamos padronizar as funções dos trabalhadores", o qual se refere ao constructo "Práticas Operacionais". Esse foi descartado por não ser relevante no contexto das grandes empresas exportadoras brasileiras, visto que todas elas já apresentam padronização em suas atividades.

Ao buscar compreender mais detalhadamente essa questão, verificou-se que há uma discrepância significativa do grau de conhecimentos dos trabalhadores em relação ao uso de EPIs e da cultura em realizar seu trabalho de maneira segura e saudável.

Para Facundes et al. (2016), ainda há uma falta de conhecimento dos trabalhadores em relação à SST e até certo desconhecimento acerca de EPI, seu uso correto e a resistência ao uso. Também há falta de adequação ergonômica dos EPIs para o gênero feminino (PONCE DEL CASTILLO, 2016). Quando o trabalho apresenta grau de periculosidade, ou seja, com risco à vida do trabalhador, a prática de uso do EPI é realizada mesmo que cause desconforto ao trabalhador, visto que a estratégia de educação continuada acerca do conhecimento dos riscos inerentes a atividade é que propicia a continuidade do uso durante a jornada laborativa (OLIVEIRA; VEIGA, 2013).

Os estudos de Cordeiro et al. (2016) demonstram que, mesmo em ambientes em que todos os colaboradores utilizam o EPI, há a percepção de que somente esses não são suficientes para prevenir a ocorrência de acidentes, fato corroborado por Monquero; Inácio e Silva (2009), que argumentam que ainda há falta de cuidado do trabalhador com a sua GEPROS. Gestão da Produção, Operações e Sistemas, v. 14, nº 2, p. 108 - 128, 2019. 
Práticas de Saúde e Segurança do Trabalho: Uma Survey em Empresas Brasileiras Exportadoras

segurança.

\subsubsection{Quanto à normalidade dos dados}

O teste de Kolmogorov-Smirnov $(\mathrm{K}-\mathrm{S})$ foi utilizado para verificar se os dados seguem uma distribuição normal. Para isso, foram criadas variáveis representantes das médias dos indicadores de cada constructo, devendo os valores "p-value" de cada média serem maiores do que $0,05(5 \%)$.

Tabela 6 - One-Sample Kolmogorov-Smirnov (K-S) Test

\begin{tabular}{llllll}
\hline Médias & ASST & SSC & CULT & QUIP4 & PRAT \\
\hline K-S teste & 0,894 & 1,204 & 0,883 & 0,950 & 0,869 \\
p-value & 0,401 & 0,110 & 0,416 & 0,328 & 0,437 \\
\hline
\end{tabular}

Fonte: Pesquisa de campo dos autores (2016).

Conforme a Tabela 6, é possível observar que todos os constructos se comportam com uma distribuição normal.

\subsubsection{Quanto à correlação dos dados}

Foi utilizada a correlação de Pearson, que mede o grau de direção, positiva ou negativa, entre duas variáveis de escala métrica. Dessa forma, a correlação foi medida entre todas as médias de cada constructo, com todas apresentando significância a um nível de $1 \%$.

Tabela 7 - Quanto à correlação entre os constructos

\begin{tabular}{llllll}
\hline & ASST & SSC & CULT & EQUIP4 & PRAT \\
\hline ASST & 1 & $0,894^{* *}$ & $0,899^{* *}$ & $0,875^{* *}$ & $0,702^{* *}$ \\
SSC & 1 & $0,887^{* *}$ & $0,796^{* *}$ & $0,823^{* *}$ \\
CULT & & 1 & $0,750^{* *}$ & $0,866^{* *}$ \\
EQUIP & & & 1 & $0,585^{* *}$ \\
PRAT & & & & & 1 \\
\hline
\end{tabular}

Fonte: Pesquisa de campo dos autores (2016).

**. Correlação significante em nível de 0,01 ou $1 \%$

Conforme Tabela 7, a maioria dos constructos apresentou uma correlação forte entre eles ( $p$ de Pearson entre 0,7 e 0,9), apenas a correlação entre os constructos "Desempenho da equipe de trabalho" e "Práticas Operacionais" se apresentou como moderada. A correlação mais forte foi entre os constructos "Ambiente seguro e Saudável do Trabalho" e "Cultura". 


\section{CONSIDERAÇÕES FINAIS}

O presente artigo buscou validar um constructo que tem como objetivo identificar como a gestão de empresas exportadoras brasileiras desenvolvem suas práticas de gestão do negócio com ênfase nas questões inerentes às práticas de saúde e segurança no trabalho (SST). O construto utilizado foi originalmente elaborado por Pagell et al. (2015) e traduzido, tendo sido alguns termos ajustados para a cultura e a língua pátria.

Concluiu-se que a escala de avaliação do nível de implementação das práticas de gestão de negócios voltadas para a saúde e segurança no trabalho (SST) pode ser validada para o contexto brasileiro.

Ao analisar os dados, buscando responder às questões previamente elaboradas para a pesquisa, antes do seu debate individualizado, observou-se que apenas $47 \%$ das empresas exportadoras analisadas concretizaram a implementação da ISO 9001. Outro fato que chamou a atenção foi que $34 \%$ delas nem sequer consideram a sua futura implementação, sendo, em grande maioria, aquelas do setor primário. Ademais, em um ambiente de manufatura, verificou-se que a maioria das empresas apresenta certificação ISO 9001.

Também, realizou-se a análise por meio do teste Kruskal Wallis, entre as variáveis cultura de segurança e saúde do trabalho e a implantação da ISO 9001. Infere-se que a implantação da certificação não garantiu uma distribuição estatística diferente para essas empresas quanto ao constructo citado.

Para os demais constructos, a implantação da ISO 9001 influencia estatisticamente o desempenho, corroborando para atender ao objetivo específico da pesquisa, o qual arguiu que o processo de implantação de um sistema de gestão agrega valor para as organizações.

Ao tentar ampliar a análise da questão para verificar se há diferença de comportamento cultural naquelas empresas que possuem certificação das normas ISO 14.000 e OSHAS 18.001, pode-se verificar que, devido à baixa implementação dessas sistemáticas normativas pelas empresas dos respondentes, não há dados suficientes para uma análise estatística com significância. Em compensação, as empresas obtiveram maiores níveis de implementação de práticas de sistema de segurança, o que demonstra a preocupação com o atendimento à legislação. A nota desse constructo reflete a visão do isomorfismo normativo gerencial típico dessa área.

A pesquisa revelou também a baixa implementação de práticas voltadas para o desenvolvimento da equipe de trabalho e sua baixa correlação com o constructo "Práticas GEPROS. Gestão da Produção, Operações e Sistemas, v. 14, nº 2, p. 108 - 128, 2019. 
Operacionais", revelando, assim, que a cultura organizacional está mais atrelada aos aspectos de infraestrutura, maquinários e legislações a serem seguidas do que propriamente aos investimentos em treinamentos e desenvolvimento de uma equipe que represente e dissemine as práticas de forma colaborativa e comunicativa.

Conclui-se, dessa forma, que a maior parte das empresas e seus funcionários estão limitados às normas, infraestrutura e processos, podendo apresentar dificuldades em desenvolver internamente essa cultura e o aprendizado em longo prazo. Como consequência, essas organizações tendem a ter um sistema de gestão instável, com produção limitada pelo número de acidentes, paradas, produtos defeituosos, entregas atrasadas e altos custos.

Quanto ao porte da empresa, questionou-se sobre a influência no nível de implantação de práticas voltadas para a saúde e segurança do trabalho. Em seguida, foram avaliadas as médias dos constructos entre o porte das empresas em relação às demais categorias, sendo possível observar que essas não apresentaram resultados significativos ou alfa maior do que $5 \%$, ou seja, o porte das empresas não influencia para que as práticas de SST sejam implementadas nas organizações.

Ao realizar a análise da confiabilidade do constructo de pesquisa, observou-se que, ao excluir algumas questões, o índice de Alfa de Cronbach apresentou melhoria. Ao avaliar os itens excluídos, observou-se que as respostas apontam que a prática já apresenta certa maturidade, portanto, sem variância.

A limitação central encontrada na realização deste estudo foi o baixo número de empresas que se prontificaram a participar da pesquisa. Embora tenha sido realizado um contato inicial telefônico e o envio do e-mail com a pesquisa a ser feita somente para aqueles que aceitaram respondê-lo, o número de retorno de respondentes foi ínfimo. A percepção dos pesquisadores durante os contatos telefônicos foi de que muitos receberam com surpresa ou desconfiança o contato acerca de uma investigação acadêmica nessa área, demonstrando muitos outros reticentes e pouco receptivos frente aos temas abordados.

Para novos estudos, sugere-se rever a estratégia de abordagem para aumentar o tamanho da amostra de empresas participantes, bem como aumentar o número de pessoas respondentes por empresa para que seja possível verificar se há discrepância interna entre as percepções dos profissionais da área da SST e as demais equipes de colaboradores. 
Práticas de Saúde e Segurança do Trabalho: Uma Survey em Empresas Brasileiras Exportadoras

\section{Referências}

ALMEIDA, C. L.; NUNES, A. B. D. A. Proposta de indicadores para avaliação de desempenho dos Sistemas de Gestão Ambiental e de Segurança e Saúde no Trabalho de Empresas do ramo de engenharia consultiva. Gestão \& Produção, v. 21, n. 4, p. 810-820, 2014.

BRASIL. Portaria $N^{\circ} 3214,08$ de junho de 1978. Normas Regulamentadoras. Ministério do Trabalho e Emprego. $1978 . \quad$ Disponível em: http://www.camara.gov.br/sileg/integras/839945.pdf . Acesso em: 06 abr. 2018.

BRASIL. Norma Regulamentadora (NR) 1 - Disposições Gerais. Ministério do Trabalho e Emprego. $2009 . \quad$ Disponível em: http://www.trabalho.gov.br/images/Documentos/SST/NR/NR1.pdf. Acesso em: 06 abr. 2018.

BRASIL. Plano Nacional de Segurança e Saúde no Trabalho. Brasília: Ministério do Trabalho e Emprego, 2012.

BRASIL. Anuário Estatístico de Acidentes do Trabalho - AEAT. 2014. Disponível em: http://www.previdencia.gov.br/dados-abertos/dados-abertos-sst/. Acesso em: 06 out. 2016.

BRASIL. Anuário Estatístico de Acidentes do Trabalho - AEAT. 2015. Disponível em: http://www.previdencia.gov.br/wp-content/uploads/2015/08/AEPS-2015-FINAL.pdf. Acesso em: 10 mai. 2017.

BROWN, K. Workplace Safety: A Call for Research. Journal of Operations Management, v.14, n.1, p. 157-171, 1996.

COOPER, D. R.; SCHINDLER, P. S. Métodos de pesquisa em administração. 10. ed. Porto Alegre: Bookman, 2011.

CORDEIRO, J. F. C.; ALVES, A. P.; GIR, E.; MIRANDA, D. O.; CANINI, S. R. M. S. Uso de equipamento de proteção individual em serviço de atenção domiciliar. Cogitare Enfermagem, v. 21, n. 3, p. 1-8, 2016.

FACUNDES, J. L.; ARANTES, T. A.; MIRANDA JÚNIOR, R. O.; BORGES, M. T. Treinamento e conscientização para auxiliar na redução de acidentes na indústria da construção civil. Revista Integração Universitária, v. 11, n. 14, p. 23-31, 2016.

FAVRETTO, J; ROMAN, D J; SEHNEM, S. Análise dos recursos impulsionadores da vantagem competitiva-o caso BRF Foods. GEPROS. Gestão da Produção, Operações e Sistemas, v. 11, n. 3, p. 153 - 168, 2016.

FENG, M.; TERZIOVSKI, M.; SAMSON, D. Relationship of ISO 9001: 2000 quality system certification with operational and business performance: A survey in Australia and New Zealand-based manufacturing and service companies. Journal of Manufacturing Technology Management, v. 19, n. 1, p. 22-37, 2007.

FILIPPE M. Como fazer. Revista Exame, São Paulo, v. 1122 n. 18, p. 140-141, 2016.

FLYNN, B. B.; SAKAKIBARA, S.; SCHROEDER, R. G. Relationship between JIT and TQM: Practices and performance. Academy of Management Journal, v.38, n.5, p.13251360, 1995.

GIL, A. C. Como elaborar projetos de pesquisa. 4. ed. São Paulo : Atlas, 2002. 
Práticas de Saúde e Segurança do Trabalho: Uma Survey em Empresas Brasileiras Exportadoras

HAIR, J. F.; ANDERSON, R. E.; TATHAM, R. L.; BLACK, W. Multivariate data analysis. New Jersey: Prentice-Hall, 1998.

HAYES, B. E.; PERANDER, J.; SMECKO, T.; TRASK, J. Measuring Perceptions of Workplace safety: development and validation of the work safety scale. Journal of Safety Research, v. 29, n. 3, p. 145-161, 1998.

INMETRO - Instituto Nacional de Metrologia, Qualidade e Tecnologia. Relatório de Certificados Válidos no Brasil ABNT NBR ISO 9001. Disponível em: http://certifiq.inmetro.gov.br/Grafico/CertificadosValidosBrasil . Acesso em: 03 março 2017.

KLINE, R. B. Principles and practice of structural equation modeling. 3rd. ed. The Guilford Press: New York, 2011.

MAEKAWA, R.; CARVALHO, M. M.; OLIVEIRA, O. J. Um estudo sobre a certificação ISO 9001 no Brasil: mapeamento de motivações, benefícios e dificuldades. Gestão e Produção, v.20, n.4, p.763-779, 2013.

MAIA, L. C. C.; ESPINDOLA, D. M.; VEIGA, C. H. A. Highlighting occupational health and safety (OHS) practices in manufacturing area: a bibliography review. RACEF - Revista de Administração, Contabilidade e Economia da Fundace. v. 7, n. 3, p. 51-63, 2016.

MALHOTRA, M. K.; GROVER, V. An assessment of survey research in POM: from constructs to theory. Journal of Operations Management, v. 16, n. 4, p. 407-425, 1998.

MDIC - Ministério de Desenvolvimento, Indústria e Comércio. Estatísticas de Comércio Exterior. Disponível em: http://www.mdic.gov.br/comercio-exterior/estatisticas-decomercio-exterior . Acesso em: Acesso em: 06 mar. 2016.

MONQUERO, P. A.; INÁCIO, E. M.; SILVA, A. C. Levantamento de agrotóxicos e utilização de equipamento de proteção individual entre os agricultores da região de Araras. Arq. Inst. Biol., v.76, n.1, p.135-139, 2009.

OLIVEIRA, M.; VEIGA, C. H. A. Segurança e saúde no trabalho em cooperativa de distribuição de energia elétrica: percepção dos colaboradores. Ação Ergonômica, v. 8, n. 2, p. 92-101, 2013.

PAGELL, M.; JOHNSTON, D.; VELTRI, A.; KLASSEN, R.; BIEHL, M. Is safe production an Oxymoron? Production and Operation Management, v. 23, n. 7, p. 1161-1175, 2014.

PAGELL, M.; KLASSEN, R.; JOHNSTON, D.; SCHEVCHENKO, A.; SHARMA, S. Are safety and operational effectiveness contradictory requirements: The roles of routines and relational coordination. Journal of Operations Management, v. 36, p. 1-14, 2015.

PAZ, F. J.; KIPPER, L. M. Sustentabilidade nas organizações: vantagens e desafios. GEPROS. Gestão da Produção, Operações e Sistemas, v. 11, n. 2, p. 85, 2016.

PINTO, S. H. B.; CARVALHO, M. M.; HO, L. L. Implementação de programas de qualidade: um survey em empresas de grande porte no Brasil. Gestão \& Produção, v. 13, n. 2, p. 191-203, 2006.

PONCE DEL CASTILLO, A. El equipo de protección individual: el desafío de la adaptación al cuerpo femenino. Laboreal, v.12, n.2, p.95-99, 2016. 
Práticas de Saúde e Segurança do Trabalho: Uma Survey em Empresas Brasileiras Exportadoras

POWER, D.; KLASSEN, R.; KULL, T. J.; SIMPSON, D. Competitive goals and plant investment in environment and safety practices: Moderating effect of national culture. Decision Sciences, v. 46, n. 1, p. 63-100, 2015.

SENRA R.; Fiscalização flagra trabalho escravo e infantil em marca de roupas de luxo em SP. Globo, 2016. Disponível em: http://g1.globo.com/economia/noticia/2016/06/fiscalizacaoflagra-trabalho-escravo-e-infantil-em-marca-de-roupas-de-luxo-emsp.html?utm_source=whatsapp\&utm_medium=share-bar-desktop\&utm_campaign=share-bar. Acesso em: 20 jun. 2016.

SHAFIQ, A.; KLASSEN, R. D.; JOHNSON, P. F. Socially Responsible Practices: Na Exploratory Study on Scale Development using Stakeholder Theory. Journal Decision Sciences, v.45, n.4, 2014.

VITORELI, G. A.; CARPINETTI, L. C. R. Análise da integração dos sistemas de gestão normalizados ISO 9001 e OHSAS 18001: estudo de casos múltiplos. Gestão \& Produção, v. 20, n. 1, p. 204-217, 2013. 